\title{
Adaptation of a U.S. evidence-based Positive Prevention intervention for youth living with HIV/AIDS in Kinshasa, Democratic Republic of the Congo
}

\author{
L. Parker ${ }^{\star}, a, 1$, S. Maman ${ }^{b, 1}$, A. Pettifor ${ }^{c, 1}$, J.L. Chalachala ${ }^{d, 1}$, A. Edmonds ${ }^{c, 1}$, C.E. Golin ${ }^{b, e, 1}$, \\ K. Moracco ${ }^{\mathrm{b}, 1}$, and F. Behets ${ }^{\mathrm{c}, 1}$ \\ aMonitoring and Evaluation Technical Advisor, Futures Group, Chapel Hill, North Carolina, USA \\ bepartment of Health Behavior, Gillings School of Global Public Health, The University of North \\ Carolina at Chapel Hill, Chapel Hill, USA \\ 'Department of Epidemiology, Gillings School of Global Public Health, The University of North \\ Carolina at Chapel Hill, Chapel Hill, USA \\ ${ }^{d}$ Kinshasa School of Public Health, Kinshasa, Democratic Republic of the Congo \\ eDepartment of Medicine, University of North Carolina at Chapel Hill School of Medicine, Chapel \\ Hill, USA
}

\begin{abstract}
Effective HIV prevention programs for people living with HIV/AIDS (PLWH) are important to reduce new infections and to ensure PLWH remain healthy. This paper describes the systematic adaptation of a U.S.-developed Evidence Based Intervention (EBI) using the Centers for Disease Control and Prevention (CDC) Map of Adaption Process for use at a Pediatric Hospital in Kinshasa, Democratic Republic of the Congo (DRC). The adapted intervention, Supporting Youth and Motivating Positive Action or SYMPA, a six-session risk reduction intervention targeted for youth living with HIV/AIDS (YLWH) in Kinshasa was adapted from the Healthy Living Project and guided by the Social Action Theory. This paper describes the process of implementing the first four steps of the ADAPT framework (Assess, Select, Prepare, and Pilot). Our study has shown that an EBI developed and implemented in the U.S. can be adapted successfully for a different target population in a low-resource context through an iterative process following the CDC ADAPT framework. This process included reviewing existing literature, adapting and adding components, and focusing on increasing staff capacity. This paper provides a rare, detailed description of the adaptation process and may aid organizations seeking to adapt and implement HIV prevention EBIs in sub-Saharan Africa and beyond.
\end{abstract}

\section{Keywords}

HIV prevention; Positive Prevention; YLWH in sub-Saharan Africa; Evidence-Based Interventions; Adaptation

\footnotetext{
(C) 2012 Elsevier Ltd. All rights reserved.

"Corresponding author at: 308 West Rosemary Street, Suite 203A, Chapel Hill, NC 27516, USA. Tel.: +1 9192404403. lparker@futuresgroup.com (L. Parker).

${ }^{1}$ For the SYMPA Study Team. See Appendix A.
} 


\section{Introduction}

Effective HIV prevention programs for people living with HIV/AIDS (PLWH) (also called "Positive Health, Dignity, and Prevention" or "Positive Prevention") are important to reduce new HIV infections and ensure that PLWH remain healthy (Global Network of People Living with HIV \& UNAIDS, 2009; Kalichman, 2005). Prevention interventions with HIVpositive persons usually have four primary goals: (1) ensure PLWH do not transmit the virus to others; (2) ensure PLWH remain physically healthy; (3) ensure PLWH remain mentally healthy; and (4) ensure the involvement of PLWH in prevention activities, leadership, and advocacy (Bunnell, Mermin, \& De Cock, 2006; WHO, 2008). In the context of Positive Prevention interventions, such as the "Seek, Test, Treat, and Retain" approach, behavioral measures to prevent sexual transmission of the virus play an important role in combination prevention strategies (Dodd, Garnett, \& Hallett, 2010; Montague, Vuylsteke, \& Buve, 2011). Furthermore, recent findings that early initiation of antiretroviral (ARV) treatment by PLWH can prevent the spread of HIV to their sexual partners are promising (Cohen et al., 2011) but will only be effective if the PLWH are adherent to their medications and if their viral loads remain undetectable. Positive Prevention behavioral interventions can reach PLWH before they are eligible for ARV treatment and can also help keep individuals engaged once they are receiving treatment (Walensky, 2009).

This paper describes the systematic adaption of a U.S. Evidence-Based Intervention (EBI), the Healthy Living Project, using the Map of Adaption Process: A Systematic Approach for Adapting Evidence-Based Behavioral Interventions Guidelines, or ADAPT, developed by the Centers for Disease Control and Prevention (CDC) for adapting evidence-based HIV behavioral programs for new settings and target populations (The Healthy Living Project Team, 1999; McKleroy et al., 2006). This paper will describe results from each step of the ADAPT guidance so that other researchers and practitioners can learn about the specific steps necessary to conduct a systematic adaptation of an EBI. These important steps are often omitted from the peer-reviewed literature because of space limitations or a preference for reporting results rather than processes. The adapted intervention was assessed for feasibility using an innovative feasibility analysis framework (Bowen et al., 2009). Findings, reported in a separate manuscript, showed that the adapted intervention was suitable, satisfying, and attractive to both program facilitators and participants, was able to be implemented effectively, performed well with a new population, and showed preliminary efficacy (Parker et al., submitted for publication).

\subsection{Youth and HIV in the DRC}

Worldwide, nearly five million youth 15-24 years of age currently live with HIV, and each year close to 900,000 youth are infected with HIV (UNAIDS, 2010). About $80 \%$ of youth living with HIV/AIDS (YLWH) or four million live in sub-Saharan Africa (UNAIDS, 2010). In the Democratic Republic of the Congo (DRC), over 60\% of the population is under 20 years of age and the prevalence of HIV among youth aged 15-24 is $0.8 \%$ overall and $1.4 \%$ in Kinshasa, the capital (Ministry of Planning Democratic Republic of the Congo, 2007).

\subsection{Positive Prevention for YLWH}

Since 2003, there have been an increasing number of studies focusing on Positive Prevention interventions with PLWH living in the U.S., including a few studies on interventions targeting YLWH (Crepaz et al., 2006). Positive Prevention may be particularly suited for YLWH, as these interventions can reach perinatally infected youth before commencement of sexual activity, and youth who acquired HIV sexually during the early stages of infection. However, Positive Prevention is a relatively new area in sub-Saharan 
Africa where many of those infected have only recently accessed care and treatment (Bunnell et al., 2006; WHO, UNAIDS, \& UNICEF, 2011). The number of YLWH in subSaharan Africa is slowly rising as these youth gain access to ARVs and live longer (WHO et al., 2011). Treatment facilities provide an important venue where YLWH receive ARVs, underscoring the opportunity for clinic-based Positive Prevention programs targeting youth to reach this underserved population.

\subsection{Adaptation of Evidence-Based Interventions}

Most Positive Prevention EBIs have been designed in the U.S. and therefore it is crucial that they be adapted systematically and appropriately prior to implementation in other settings. Adaptation is important to ensure that the intervention is appropriate and relevant for the target population as well as the needs and capacities of the implementing organization (McKleroy et al., 2006). While there is no assurance that once an intervention is adapted it will continue to show efficacy, a significant number of HIV prevention EBIs have maintained efficacy after undergoing the adaptation process (Gaydos et al., 2008; Kelly et al., 2000;Lightfoot, Kasirye, Comulada, \& Rotheram-Borus, 2007; Vanden-houdt et al., 2010; Wu et al., 2003). It is important to acknowledge that there are significant challenges in adapting and implementing EBIs in non-research settings (Bell et al., 2007; Dolcini et al., 2010; Morrison et al., 2009). However, when attention is given to maintaining core elements and ensuring sufficient capacity for adaptation and implementation, adapted interventions have a greater chance of success (Galbraith et al., 2009).

\subsection{Adaptation of Positive Prevention interventions in sub-Saharan Africa}

A few Positive Prevention EBIs predominantly targeted to adults living with HIV/AIDS have recently been adapted for implementation in sub-Saharan Africa. Healthy Relationships and WiLLOW, both group-based interventions for adults living with HIV/AIDS, have been adapted in South Africa (Cloete et al., 2009; Saleh-Onoya et al., 2009). The Healthy Relationships intervention focuses on three areas: disclosure to sex partners, disclosure to family and friends, and HIV transmission risk reduction. Through group discussions, role plays, videos, and skill-building exercises, the intervention aims to help PLWH develop skills to cope with HIV-related stressors and risky sexual situations (Cloete et al., 2009; Kalichman et al., 2001). Preliminary results from the pilot adaptation of Healthy Relationships showed that participants were more confident in using a condom after participating in the intervention (Cloete et al., 2009). WiLLOW is a peer-led intervention targeting HIV-positive African American women that emphasizes gender pride and focuses on self-esteem, relationships, and sexual health (Wingood et al., 2004). While the WiLLOW intervention reported lower incidence of sexually transmitted infections (STIs) for the intervention group, the intervention and control groups did not differ on coping or condom use skills (Saleh-Onoya et al., 2009).

Provider-delivered interventions such as Partnership for Health and Options have been adapted for implementation in clinics in sub-Saharan Africa (CDC, National Center for HIV/ AIDS Viral Hepatitis STD and TB Prevention, \& Global AIDS Program, 2008; Dewing et al., 2010; Evangeli et al., 2009; Peltzer, Tabane, Matseke, \& Simbayi, 2010). Partnership for Health is a brief, provider-delivered counseling program for PLWH that focuses on improving patient-provider communication about safer sex, disclosure of serostatus, and HIV prevention (Richardson et al., 2004). A multi-site evaluation is currently being conducted in Kenya, Namibia, and Tanzania but no papers have been published to date (Bachanas, 2011; CDC et al., 2008). Options is an intervention based on motivational interviewing techniques whereby providers (1) evaluate the risk behaviors of their HIVpositive patients and assess the readiness of patients to change risky behaviors, if necessary and (2) support the patients through this process (Fisher et al., 2006). Options has been 
adapted and implemented in multiple sites across South Africa with mixed results. All of the adapted interventions used counselors instead of providers to deliver the intervention. One study of 152 HIV-positive patients over the age of 18 reported a decreased number of unprotected sex acts for participants in the intervention arm compared to the control arm (Cornman et al., 2008). This same adaptation was implemented at 13 public HIV counseling and testing sites in South Africa $(n=488)$ and was found to significantly reduce multiple partners, unprotected sex, alcohol or drug use in a sexual context, and transactional sex, even though the average number of sessions attended by each patient was only 2.3 out of three sessions (Peltzer et al., 2010).

Another adaptation implemented by a non-governmental organization serving peri-urban communities in the Western Cape Province of South Africa evaluated the effectiveness of training lay counselors $(n=14)$ in the motivational interviewing techniques used in the Options intervention and found that only three of the 14 counselors achieved beginning proficiency in the techniques (Evangeli et al., 2009). Likewise, a case study of one lay counselor implementing the Options intervention in an ARV clinic in Cape Town, South Africa reported that the intervention was not delivered with fidelity and less than one third of intended recipients received the intervention (Dewing et al., 2010).

Only one EBI, Together Learning Choices (TLC), an intervention for YLWH that focuses on enhancing health behaviors, increasing condom use, eliminating or reducing unprotected sex, and eliminating or reducing drug and alcohol use, has been adapted for YLWH in Uganda (Lightfoot, Kasirye, et al., 2007; Rotheram-Borus et al., 2001). YLWH who participated in the 18-session intervention $(n=50)$ reported more consistent condom use and reduced numbers of sexual partners compared with the control group $(n=50)$ (Lightfoot, Kasirye, et al., 2007).

In summary, while promising, adaptations of EBIs in sub-Saharan Africa have faced challenges related to recruitment and retention, mastery of skills by counselors, connection of outcomes with the intervention, and achieving targeted behavioral changes. The challenges these adaptations reported provided lessons that were applied during this study. Furthermore, the majority of adapted interventions have been implemented with adult populations and thus there is a need to adapt Positive Prevention EBIs for YLWH. The one intervention adapted for YLWH was delivered to individuals during home visits by nurses; therefore, there is a need to determine the effectiveness of interventions delivered through different delivery modes that are less resource-intensive, such as group settings or during routine clinic visits. In addition, while there are some examples in the literature that detail the intervention adaptation process, very few have been conducted in low-resource contexts (Poulsen et al., 2010; Saleh-Onoya et al., 2008; Wainberg et al., 2007), with only one in subSaharan Africa (Saleh-Onoya et al., 2008).

\section{Methods}

\subsection{Study overview}

This study was part of a larger project with the aims of adapting, implementing, and conducting a feasibility study of an evidence- and clinic-based Positive Prevention intervention with YLWH 15-24 years of age in Kinshasa, DRC. The goal of the project is to develop a Positive Prevention program that addresses the youths' developmental needs and helps them cope with HIV in the context of their emerging independence and sexuality. The central purpose of the larger study was to determine whether the incorporation of evidencebased Positive Prevention into the standard of care is feasible for both the target population of YLWH and the implementing organization. This paper will describe the systematic adaptation process used as well as lessons learned from the adaption process. 


\subsection{Study site}

A family-centered HIV care and treatment program at a pediatric hospital in Kinshasa was the site of this study. The program provides comprehensive HIV medical care including ARV treatment and psychosocial support to children and their parents or guardians, as well as other household members. As of April 2012, the program had served more than 2146 HIV-positive patients and currently following 191 YLWH aged 15-24. The adapted intervention was implemented with young men $(n=6)$ and young women $(n=7)$ between 15 and 20 years of age (Parker et al., submitted for publication).

\subsection{Theoretical framework for adaptation}

The ADAPT guidelines are based largely on Rogers' Diffusion of Innovations Theory and also include principles of community health education, social work, participatory research, and community empowerment (McKleroy et al., 2006; Rogers, 1995). The CDC ADAPT guidance defines adaptation as "The process of modifying key characteristics of an intervention, recommended activities and delivery methods, without competing with or contradicting the core elements, theory, and internal logic of the intervention thought most likely to produce the interventions' main effects" (McKleroy et al., 2006). Core elements are those "most likely to lead to the intervention's main effects" while key characteristics are "important but not essential attributes of an intervention's recommended activities and delivery methods" (McKleroy et al., 2006). The internal logic is the explanation of "relationships among activities, behavioral determinants, and the intended outcome(s)" (McKleroy et al., 2006). The guidelines include five key steps: (1) Assess, (2) Select, (3) Prepare, (4) Pilot, and (5) Implement.

This tool was selected because it is specific to HIV prevention, developed by the CDC which is responsible for designating HIV prevention interventions as evidence-based, and was the most user friendly of the different adaptation tools considered (Card, Solomon, \& Cunningham, 2011; Tortolero et al., 2005; Wainberg et al., 2007; Wingood \& DiClemente, 2008). We followed the first four steps of the ADAPT framework for this study. The steps were followed as closely as possible given the resource, organizational capacity, and time constraints. The first step, Assess, includes evaluating the target population, the potential EBIs, and the implementing organization's capacity. The second step, Select, involves the decisions related to whether the EBI will be adapted or implemented as is, or whether a different intervention all together will be selected. The third step, Prepare, includes adapting intervention materials and curriculum as well as pre-testing these materials, increasing agency capacity, and developing necessary partnerships. The fourth step, Pilot, involves pilot testing the components of the adapted intervention and developing an intervention plan. The fifth step, Implement, involves implementing the entire adapted intervention. Finally, it is important to note that the guidance includes feedback loops among the different steps and many of the steps can be conducted simultaneously rather than sequentially (McKleroy et al., 2006). The section that follows will describe the results of each of the steps of the adaptation process.

\section{Results}

\subsection{Action step 1: assess}

3.1.1. Target population-The target population was YLWH between the ages of 15-24 seeking care at the clinic in Kinshasa, DRC. Prior to the adaptation process, preliminary formative research was conducted with approximately 200 YLWH ages 14-24 who were clients of four HIV treatment, testing, or counseling centers in Kinshasa (Behets \& Pettifor, 2006). That research was conducted to explore sexual behaviors, knowledge of HIV prevention, health seeking behaviors, psychosocial wellbeing, alcohol and drug use, and 
attitudes, perceptions, and norms around sexuality (including marriage and child-bearing) and HIV prevention amongst YLWH (Behets \& Pettifor, 2006). The research found that over half of the young women and a quarter of the young men had already had sexual intercourse, with only $53 \%$ of young women and $67 \%$ of young men reporting condom use at their most recent sexual act (Pettifor et al., 2008). In addition, just over a third of the young women and only a quarter of young men reported having disclosed their HIV status to their most recent sexual partner. These results showed that this population was at risk of transmitting the virus to their partners through unprotected sex and indicated the importance of Positive Prevention programs for YLWH in this environment (Morin et al., 2007). All youth surveyed in the formative research expressed interest in Positive Prevention topics including: making decisions about condom and contraceptive use, how to protect against HIV infection, and how to make life better overall (Behets \& Pettifor, 2008).

Information from this study was analyzed in combination with results from an in-depth literature review in order to determine the risk behaviors and their determinants in this specific population. In addition, the study team reviewed relevant theories and their common theoretical constructs and evaluated these constructs based on their relevance and changeability using Intervention Mapping as a guide (Bartholomew, Parcel, \& Kok, 1998; Noar, 2007). Furthermore, the study team assessed empirical data from previous research with YLWH in both the U.S. and sub-Saharan Africa. After this process the research team decided that the intervention would focus on the following six determinants of high risk sexual behavior for YLWH: lack of HIV/STI knowledge, low condom use skills, weak coping mechanisms, limited ability in sexual communication/negotiation, lack of disclosure, and low levels of social support. It was determined that the best location for an intervention with this target population would be the clinic, as this was an easily accessible, central location where all YLWH were comfortable visiting. The intervention was scheduled to take place on the weekend so that it would not interfere with the schooling of the YLWH.

3.1.2. Interventions-Next, potential EBIs that could be adapted for this population were identified. Four EBIs for Positive Prevention were selected to be considered for adaptation: Healthy Relationships, CLEAR, TLC, and The Healthy Living Project (HLP) (Kalichman et al., 2001; Rotheram-Borus, Kelly, et al., 2004; Rotheram-Borus et al., 2001; The Healthy Living Project Team, 2007). These four interventions were chosen based on the extent to which they incorporated the risk behaviors and their determinants identified during the assessment of the target population. Interventions focusing only on women or gay/bisexual men or using only peer counselors or health care providers during routine office visits were not selected. Each of the four EBIs identified were compared based on their core elements, key characteristics, delivery methods, and theoretical bases. This information was obtained from peer-reviewed journal articles and implementation manuals publically available and through contact with research authors (AED, 2011; CDC, 2012a, 2012b; Rotheram-Borus, 1999, 2001; The Healthy Living Project Team, 2004).

When examining the selected EBIs, we noted that they shared many of the same core elements and key characteristics identified during the CDC best evidence intervention selection. Delivery methods for the four EBIs were also similar, with all four using exercises, goal setting, and role plays. All four EBIs incorporated elements of the Social Cognitive Theory (Bandura, 1989), while TLC and the HLP were also based on Social Action Theory (SAT) (Ewart, 1991). Finally, we considered the resources and costs necessary for implementing each program, although only the 23-session group intervention TLC had published cost-effectiveness data available. The cost of implementing TLC in four urban areas of the U.S. was $\$ 522$ per YLWH and the authors estimated that over three months, the intervention could prevent two new infections per 1000 participating YLWH (Lee, Leibowitz, \& Rotheram-Borus, 2005). 
3.1.3. Goodness of fit-The risk behaviors and behavioral determinants addressed in each of the EBIs were examined to determine the extent to which they matched the determinants identified in our target population. Healthy Relationships, TLC, CLEAR, and the HLP addressed almost all of the same risk behaviors and behavioral determinants. However, Healthy Relationships had a much more pronounced focus on the lack of selfefficacy for disclosure of serostatus. Although this was one of the six determinants identified for the target population for YLWH in Kinshasa, it was apparent in the formative research that other determinants may play a larger role in the population and needed to be considered equally.

CLEAR, TLC, and the HLP all included goal setting, problem solving, and improved communication/assertiveness as core elements, while the HLP also included coping skills and disclosure. The Healthy Relationships core elements included primarily coping skills and disclosure as well as using movie clips and role-plays. The TLC intervention appeared to combine multiple different determinants and behaviors in the same module and thus there was not a meaningful progression of activities throughout the intervention. Although CLEAR better organized its sessions and activities, the entire first module of CLEAR focused on substance abuse, and substance abuse was highly integrated within the sexual behavior module as well. As substance abuse was not determined to be an issue for the target population, CLEAR did not appear to be a good fit.

Surprisingly, as TLC and CLEAR were developed for youth, these interventions used much more sexually explicit language than the HLP. In addition, the HLP sessions provided case studies specific to persons who were not sexually active. Because the target population for KLL consisted both of youth who had never had sex and those who had, this was important to consider. The HLP appeared to be the most comprehensive intervention, and the flow and progression of sessions and activities was better organized by topic than the other interventions assessed. In addition, even though the HLP was developed for use with adults, its specific session activities appeared to be more user-friendly and easily adaptable than the other interventions' sessions.

These interventions shared many of the same key characteristics. In at least two of the EBIs being assessed, the following key characteristics were included: incentives, limited session times, time between sessions, and use of experienced facilitators. Delivery methods for the four EBIs were also similar, with all four using exercises, goal setting, and role plays.

Healthy Relationships and CLEAR used lectures, CLEAR and TLC used skill practice, and CLEAR and the HLP used problem solving. Both Healthy Relationships and TLC used video as well as written materials. Finally, TLC and Healthy Relationships were groupdelivered while CLEAR and the HLP were delivered one-on-one (see Table 1).

3.1.3.1. Preliminary areas where EBIs need adaptation: Though it would be ideal to implement an intervention covering all of the relevant risk behaviors included in the EBIs, the study team felt that this would not be feasible in a resource-deprived setting such as the DRC. Therefore, regardless of which EBI was selected, it would need to be significantly pared down to focus on risk behaviors that were most relevant for YLWH in Kinshasa. Likewise, we decided that the extensive use of video in the adapted intervention would be difficult because culturally relevant and language-specific videos were not available.

3.1.4. Organizational capacity: resources and experience-Next, an analysis of the capacity of the clinic to implement the pilot intervention was conducted. First, after an extensive review of the intervention materials for each of the EBIs, we assessed the resources necessary to implement each of the EBIs, including program staff supplies, space, and funding for participant incentives. Second, we evaluated the extent to which the 
organization had access to the target population of YLWH as well as the program's fit with the clinic's mission. Through informal discussions with program staff and administrators, we identified gaps in experience of the organization and personnel regarding the target population, implementation, monitoring, analysis, fiscal accountability, and specific intervention skills necessary for adaptation and implementation of an EBI at the clinic. Program staff, who had been working at the clinic for an average of three and a half years, had extensive and adequate experience conducting both qualitative and quantitative research as well as providing HIV medical and psychosocial services to the YLWH target population.

Gaps were identified in the areas of advanced facilitation and counseling skills for peer groups, implementation of EBIs, and data analysis. In many of the EBIs considered, the sessions were facilitated by individuals who held degrees in psychology or social work. In this setting, requiring these qualifications would have made routine implementation of the intervention impossible, if it was found to be effective. In addition, most providers at the clinic lacked extensive experience or training in Positive Prevention. Finally, none of the program staff had implemented a multiple session prevention intervention or analyzed intervention data. Therefore, it would be necessary to strengthen these areas before implementation.

3.1.5. Stakeholders-As the final part of step 1, Assess, existing and potential stakeholders and areas of capacity building were identified. Research staff as well as administrative providers at the clinic were consulted, and their input was incorporated into the intervention planning. One community-based HIV/AIDS care and treatment center serving YLWH, AMO Congo, and two HIV support centers, Fondation Femme Plus and EVREJ-CONGO participated in the preliminary formative research. As a result, relationships between the program and these organizations were adequate. Finally, the research team attempted to locate competing programs in order to prevent any overlap, but none were identified.

\subsection{Action step 2: select}

\subsubsection{Decide to adopt, adapt, or select another intervention}

After the extensive analyses in step 1, Assess, the HLP appeared to address the expressed needs of YLWH in Kinshasa and their caregivers and be suitable to adaptation for a youth population in a low-resource setting. It was selected to be adapted (The Healthy Living Project Team, 2007).

3.2.1.1. The Healthy Living Project-The HLP is an individual Positive Prevention intervention targeting adults living with HIV in the U.S. Developed by Craig Ewart in the early 1990s and updated in 2009 and is guided by Social Action Theory (Ewart, 1991, 2009). The theory purports that "health-related behavior is bound to the social environment in which we live. In order to change behavior one must appraise a behavior to require changing, must develop self-regulatory processes (such as coping, social support, and stress management) that facilitate changing that behavior, and may need to address environmental influences that promote the risk behavior. One must also work to maintain behavior change by reinforcing relevant skills and generalizing new skills to different situations" (RotheramBorus, Swendeman, et al., 2004).

The HLP intervention uses individually tailored sessions to develop a "Life Project" which addresses quality of life, coping, methods to realize a positive healthy action state, and the development of social support relationships (The Healthy Living Project Team, 2007). Over three modules of five sessions each, participants learn skills necessary for the reduction of high risk sexual behavior (see Table 2). Participants are asked to identify their own high risk 
behavior triggers in order to help them develop mechanisms to modify these high risk behaviors. The program helps increase self-efficacy to use condoms, and teaches skills to negotiate condom use. It also teaches participants how to disclose their HIV status to their partners. Finally, access to health services, adherence to ARVs, and increased participation in medical decision making are also covered with the participants.

\subsection{Action step 3: prepare}

\subsubsection{Make necessary changes to EBI}

Next, the study team identified areas where the selected EBI needed to be adapted, and made the necessary changes to the EBI ensuring that core elements and key characteristics were maintained to the extent possible (see Table 3). First, we changed the name of the intervention from the HLP to Supporting Youth and Motivating Positive Action, or SYMPA (meaning "cool" in French), to make it more relevant for the target population. Second, the HLP was originally implemented on an individual basis. However, in a low-resource setting like Kinshasa, having multiple, individual sessions was not feasible and could potentially limit wider implementation of the intervention. In this setting, YLWH already met for monthly peer educational sessions and expressed in formative research that they enjoyed meeting in groups with their peers (Behets \& Pettifor, 2006). After reviewing the activities in the HLP curriculum, we determined that it would be feasible to adapt the majority of activities for a group setting. Thus, for these activities, rather than youth working independently, adaptations were planned to modify the activities so that youth could incorporate feedback from both facilitators and their peers so that completing these activities becomes a collaborative effort. Therefore, the intervention was adapted to be delivered in a group setting.

Third, we reduced the number of sessions from 15 to six, a number that was more realistic to implement in this setting while still able to effectively cover the primary determinants of high risk sexual behavior identified for the target population. This was done by combining similar sessions and eliminating content not directly relevant for the target population. For example, the original module three of the HLP focused on accessing health services, adherence, and active participation in medical care decision making. As these sessions did not reflect the identified risk behaviors and their determinants for the target population, the first four sessions of this module were not included in the adapted intervention (The Healthy Living Project Team, 2007). Likewise, stress and coping was originally covered over two sessions but was combined into one session for the adapted intervention. In addition, the disclosure session was combined with the social support session and the safer behavior session was combined with the assertive communication session. Finally, the last session of the original module three was included in the adapted intervention as the final session, as there was an important emphasis on maintaining behavioral change after the end of the intervention. The process of eliminating content was more straightforward than combining content from different sessions, as there was no guiding mechanism to determine exactly which activities should be combined with other activities. However, the final sessions reflected the overall content and flow of the HLP and appeared to be appropriate for the new target population.

Fourth, as study staff reported that in Congolese society sexuality is not traditionally discussed in mixed gender groups, it was decided that the groups would be separated by sex - one for young women and the other for young men, so that participants would be comfortable discussing sensitive issues. Fifth, the original intervention was targeted to PLWH who also had substance abuse problems. Substance abuse was not determined to be a problem for most YLWH at the clinic during the formative research. Therefore, the curriculum was adapted so that references to substance abuse were taken out. Sixth, the 
original intervention was targeted toward an adult population; thus, the adapted intervention incorporated specific role plays better targeted for a youth population, and references to adult issues such as work were replaced with school. Likewise, certain activities such as the condom demonstration and the STI activities were simplified to aid the youth in understanding the instructions.

Seventh, after extensive discussion, study staff determined that the intervention would target youth thought to have already been sexually active, since the original intervention was intended for a sexually active adult population. However, staff thought that youth might not be open and honest about their previous sexual activity if asked related questions in a screening form, and therefore activities were adapted to incorporate the possibility that youth had not yet had sex. For example, for YLWH who had not yet had sex, activities allowed the youth to consider issues around sexual risk once they became sexually active. Abstinence was included as an option for those youth who were already sexually active. Eighth, the entire curriculum was translated into French, and specific activities requiring YLWH to complete forms were translated into the colloquial Lingala used in Kinshasa. Ninth, a large part of module three of the HLP focused on adherence to ARVs while formative work showed that this topic was already being adequately addressed at the clinic through psychosocial counseling. Thus, the components of the original intervention focusing on medication adherence were eliminated.

Finally, tenth, an important adaptation to the intervention, the entirely new Very Important Person (VIP) component, was added to help provide support to YLWH over the long term in achieving their goals and objectives developed during the intervention. Adaptation of an intervention can include modifications to content including deletion, changes, and additions; however, researchers should ensure that modifications made do not alter the core elements and key characteristics (Galbraith et al., 2009). The VIP is an individual the YLWH identifies as a trusted person to whom he/ she would be willing to or has already disclosed his/her HIV status, and to whom the YLWH would go to for support. YLWH were asked to identify a VIP who could aid them in maintaining lower risk sexual behavior practices and staying healthy after the program ends. In conjunction with their VIP, youth were asked to identify resources and skills to support their plan to reduce risk behaviors and improve coping and problem solving skills. Given that many YLWH in the population had only disclosed their HIV status to one person, having someone who knows their status, who is supportive of them in numerous ways, and whom the youth respects, can confide in, trust, and rely on may be the key to YLWH obtaining the social support necessary to help them make healthy decisions (Luthar, Sawyer, \& Brown, 2006).The final topics of the adapted six sessions include: (1) Living with HIV, (2) Coping and Problem Solving, (3) Safer Sex I, (4) Safer Sex II, (5) Social Support and Disclosure, and (6) Wellbeing and Health Maintenance. A description of each of these sessions is included in Table 4. Finally, a conceptual model based on Social Action Theory was adapted to reflect the specific contextual influences, self-change processes, and action states to incorporate elements specific to the target population (Ewart, 1991; Lightfoot, Rotheram-Borus, \& Tevendale, 2007). The adapted conceptual model was developed for the SYMPA intervention so that the processes through which behavior change occurs amongst YLWH were clearly designated and understood by both the researchers and intervention staff (Fig. 1).

\subsubsection{Organization preparation}

A Congolese medical doctor working for the research program was chosen to serve as the Kinshasa-based program coordinator for the intervention study, and worked under the supervision of the U.S.-based study coordinator. One male and three female facilitators were recruited. Three were psychosocial counselors and one was a nurse with training in family planning. The facilitators had been working at the clinic for between seven months and six 
years. One male and one female would facilitate the young men's group, while the two females would facilitate the young women's group. The roles and responsibilities for each member of the study team were clearly laid out in eight Standard Operating Procedure (SOP) documents.

\subsubsection{Pre-test}

Next, the intervention materials were pre-tested. A half-day adaptation workshop was conducted during which members of the study team and key providers and administrators at the HIV care and treatment clinic were invited to review the intervention materials to further tailor the program for usability and cultural relevance. Each session of the program was presented and cognitive interviewing techniques with "think aloud" reactions to each activity were used for assessment. During part of this adaptation workshop, the cultural and social factors that are important to the study population were discussed and participants made suggestions to remove information or activities that they felt were not relevant.

The VIP component raised important confidentiality concerns among staff and became the primary topic explored during the adaptation workshop. Adaptation workshop participants discussed the role that the VIPs play in the lives of YLWH as well as the perceived challenges in incorporating VIPs into the SYMPA intervention. Adaptation workshop participants were concerned about confidentiality, recruitment, and the issue of who gets to choose the VIP (i.e., the facilitator or the youth themselves). We also discussed the types of skills required of VIPs necessary for sustaining the healthy behaviors and mental wellbeing of the YLWH after the end of the program. It was decided that the VIPs would be included in the final session and that confidentiality would be stressed to youth throughout the intervention. Additionally, although it was decided that the YLWH would have the final say regarding his or her VIP choice, the facilitator was encouraged to provide advice and support throughout the decision making process.

\subsubsection{Training}

A two-day training on the SYMPA intervention was held with the Kinshasa-based program coordinator and the four facilitators. Before reviewing the curriculum, fundamental aspects of Positive Prevention were covered in order to increase the capacity of the program staff for effective implementation of the intervention. During the training, each session was reviewed in detail and each activity was practiced as a group so that the facilitators became confident in their knowledge and understanding of intervention content as well as their ability to conduct each activity. Small adjustments were made to specific activities to aid comprehension for the YLWH. Additionally, role plays were further adjusted to ensure cultural relevance and to match the developmental level of youth. This training provided a forum for the facilitators to learn how to exchange ideas about Positive Prevention with YLWH, and to learn from each other and the trainer.

\subsection{Action step 4: pilot}

\subsubsection{Implementation plan for adapted EBI}

After the pre-test, training, and final adaptations were made to the intervention, a plan was developed for piloting the adapted intervention. This plan included a timeline for recruitment, implementation, data collection, and analysis as well as an outline of the key activities to be conducted for each of these phases. At this time, the final program budget was developed and approved. The SOPs were finalized and discussed further with all program staff. Communication protocols were established and final preparations for implementation were conducted. It was determined that the intervention would be piloted with two single-sex groups with between five and ten participants each. Each session was 
planned to last approximately 90 min with the six sessions spread over a period of six weeks.

A recent examination of the feasibility of public health interventions led to the development of guidelines to help evaluate and prioritize those interventions deemed "feasible" and that have the greatest likelihood of being efficacious (Bowen et al., 2009). These guidelines served as the framework for the analysis of the study data collected prior to, during, and after the implementation of the adapted pilot intervention. For the purposes of the pilot study, data were collected on the following four areas of the Bowen Feasibility Framework: acceptability, implementation, adaptation, and limited-efficacy testing. The results of the analysis of the pilot test of the SYMPA intervention will be reported in an upcoming manuscript (Parker et al., submitted for publication).

\section{Lessons learned}

Throughout the structured adaptation, we faced key challenges and learned important lessons. The first challenge was implementing an EBI in a very different setting from where the original EBI was implemented, both culturally and geographically, with very little existing guidance or literature. This challenge was overcome by closely adhering to the ADAPT process which allowed for changes to intervention elements so that the adapted intervention would be appropriate for this unique context. The study team learned that the quality of work conducted during the first step of the ADAPT framework, Assess, determines the success of the future steps. Extensive preliminary studies conducted with YLWH in Kinshasa prior to this adaptation process allowed the researchers to more accurately adapt specific components of the intervention content as well as logistics related to implementation. Likewise, exhaustive assessment of the core elements, key characteristics, and delivery methods of the Positive Prevention EBIs allowed the research team to become familiar with Positive Prevention intervention core principles and processes (Rotheram-Borus, Ingram, Swendeman, \& Flannery, 2009; Rotheram-Borus, Swendeman, \& Chovnick, 2009). In addition, extensive review of the candidate EBIs theoretical foundations, content, sessions, and activities permitted research staff to make the best decision for the targeted population.

The second challenge faced was the lack of existing Positive Prevention EBIs that targeted YLWH. However, the study team felt that Positive Prevention interventions targeting adults living with HIV addressed similar theoretical constructs and behavioral determinants. Therefore, an important lesson learned for study staff was that it is not necessary to use an EBI that targets the exact same population. However, extensive modifications were required as part of the adaptation process to ensure that intervention content was relevant and appropriate for YLHA 15-24 years of age.

Third, a related challenge was the paucity of published data on the cost-effectiveness of existing Positive Prevention EBIs. This meant that assessing the goodness of fit of the selected EBIs was difficult especially because in a resource-constrained environment such as the DRC considerations of cost are of utmost importance. The study team chose not to rely extensively on technology and the number of sessions was significantly reduced to address resource constraints. Likewise, the research team remained cognizant that only those adaptations that could be feasibly implemented within the existing health structure using existing resources could be included. Therefore, the study team learned that an EBI previously implemented successfully in a high-resource country can be suitably adapted in a low-resource country.

Fourth, before the research began, the study team had limited experience adapting or implementing structured programs or EBIs and this was a significant potential challenge in 
successful implementation. However, the process of adaptation not only aided in adapting the intervention, but also served as training in concepts and strategies for implementing HIV prevention research, particularly for the intervention facilitators. Therefore, the study team became aware that the process of adapting the intervention itself potentially increased the capacity of the staff to effectively implement the intervention.

Fifth, as the study team moved through the steps of the adaptation framework, it became evident at certain points in the process that it would be helpful to repeat some components of earlier steps. For example, during the adaptation process, the study team felt that it was important to assess the capacity of the organization not only during the preliminary step but also during all subsequent steps so that gaps in capacity could be addressed as they arose. This was initially seen as a challenge in the process, as capacity assessment can be a timeintensive process. However, the study team came to understand that adaptation is an iterative process - the feedback loops between steps are crucial to ensure that appropriate decisions are being made and that the eventual adapted intervention is successful.

Sixth, an important challenge faced by the study team was that none of the existing EBIs incorporated caregivers into the intervention. Preliminary studies showed that YLWH reported feeling isolated in their daily lives and received very little psychosocial support outside of the clinic (Behets \& Pettifor, 2008). Likewise, caregiver support is believed to be an important factor for helping YLWH maintain the success of any Positive Prevention intervention (Holub, 2010). Therefore, the study team felt this aspect needed to be incorporated into the final adapted intervention. The study team learned from this challenge that the adaptation process needed to be flexible enough to allow the addition of new content when necessary to address specific needs not included in the original intervention. Thus, the VIP component was added to the final adapted intervention in order to bolster the existing curriculum and to provide YLWH with new systems of support. The addition of the VIP component also ensured a greater probability of sustaining any positive behavior changes the YLWH made as a result of the intervention. Finally, by incorporating caregivers into the intervention, the reach of the health messages in the adapted intervention grew wider and support of participation by YLWH in the intervention increased. Consequently, the study team learned that it is not only appropriate to add components to the original intervention, but also that by doing so the intervention may be strengthened overall. All of these challenges described led to the development of key lessons learned that other researchers may find useful in their own adaptations of EBIs in different contexts.

\section{Discussion}

This study was the first to use the CDC ADAPT framework to adapt a Positive Prevention EBI for implementation with YLWH in sub-Saharan Africa. This paper described the process of following the first four steps in the ADAPT framework: (1) Assess, (2) Select, (3) Prepare, and (4) Pilot. The results from step four, Pilot, will be used to further adapt the intervention for larger-scale implementation in order to complete the ADAPT framework with Action Step 5: Implementation of the Adapted EBI. This study has shown that EBIs developed and implemented in the U.S. can be adapted successfully for a different target population in a low-resource context through an iterative process following the CDC ADAPT framework. This process included carefully reviewing the existing literature, adapting and adding components as necessary, and focusing on increasing staff capacity throughout the adaptation process. This paper provides a rare detailed description of the adaptation process and may aid other organizations that seek to adapt and implement other HIV prevention EBIs in sub-Saharan Africa or beyond. 


\section{Acknowledgments}

The HIV care and treatment program in Kinshasa has received funding or support from multiple donors including the CDC Global AIDS Program originally as part of the University Technical Assistance Program; Providing AIDS Care and Treatment in the DRC under the President's Emergency Plan for AIDS Relief; the William J. Clinton Foundation; the Elizabeth Glaser Pediatric AIDS Foundation; the Global Fund to Fight AIDS, Tuberculosis, and Malaria; the United Nations Children's Fund; and the Belgian Cooperation. The first author has received funding from the Ruth L. Kirschstein National Research Service Institutional STD/HIV Pre-Doctoral Training Award (National Institutes of Health) through the University of North Carolina at Chapel Hill Institute for Global Health \& Infectious Diseases. The authors would like to sincerely thank the health care providers who participated in this study for the selfless work they do each and every day to help young people living with HIV in the DRC.

\section{Appendix A}

\section{SYMPA Study Team:}

Delphine Kizungu, Kinshasa School of Public Health, Kinshasa, The Democratic Republic of Congo.

Alice Tabala, Kinshasa School of Public Health, Kinshasa, The Democratic Republic of Congo.

Clarisse Bokwala, Kinshasa School of Public Health, Kinshasa, The Democratic Republic of Congo.

Sammy Siwadio, Kinshasa School of Public Health, Kinshasa, The Democratic Republic of Congo.

Jeanne Ngwele, Kinshasa School of Public Health, Kinshasa, The Democratic Republic of Congo.

Odette Daiku, Kinshasa School of Public Health, Kinshasa, The Democratic Republic of Congo.

Faustin Kitetele, Kinshasa School of Public Health, Kinshasa, The Democratic Republic of Congo.

Cathy Akele, Kalembe Lembe Pediatric Hospital, Kinshasa, The Democratic Republic of the Congo.

\section{References}

AED. DEBI: Diffusion of Effective Behavioral Interventions. 2011. Retrieved from: http:// www.effectiveinterventions.org/en/HighImpactPrevention/Interventions.aspx

Bachanas P. Personal communication. 2011

Bandura A. Human agency in social cognitive theory. American Psychologist. 1989; 44(9):1175-1184. [PubMed: 2782727]

Bartholomew LK, Parcel GS, Kok G. Intervention mapping: A process for developing theory and evidence-based health education programs. Health Education \& Behavior. 1998; 25(5):545-563. [PubMed: 9768376]

Behets, F.; Pettifor, A. Formative research to develop effective prevention interventions in HIV+ youth in Kinshasa: Research study protocol. Chapel Hill: The University of North Carolina; 2006.

Behets, F.; Pettifor, A. Preventing HIV transmission and promoting psychosocial wellbeing among HIV+ youth: R01 grant application submitted to the NIH. Kinshasa, Democratic Republic of the Congo: The University of North Carolina Chapel Hill; 2008. 
Bell SG, Newcomer SF, Bachrach C, Borawski E, Jemmott JB, Morrison D, et al. Challenges in replicating interventions. Journal of Adolescent Health. 2007; 40(6):514-520. [PubMed: 17531757]

Bowen DJ, Kreuter M, Spring B, Cofta-Woerpel L, Linnan L, Weiner D, et al. How we design feasibility studies. American Journal of Prevention Medicine. 2009; 36(5):452-457.

Bunnell R, Mermin J, De Cock KM. HIV prevention for a threatened continent: Implementing positive prevention in Africa. Journal of the American Medical Association. 2006; 296(7):855-858. [PubMed: 16905790]

Card JJ, Solomon J, Cunningham SD. How to adapt effective programs for use in new contexts. Health Promotion Practice. 2011; 12(1):25-35. [PubMed: 19858321]

CDC. Best-evidence interventions. 2012a. Retrieved from: http://www.cdc.gov/hiv/topics/research/prs/ subset-best-evidence-interventions.htm

CDC. Healthy relationships intervention resources. 2012b. Retrieved from: http:// www.effectiveinterventions.org/en/Interventions/HealthyRelationships.aspx

CDC. National Center for HIV/AIDS Viral Hepatitis STD and TB Prevention, \& Global AIDS Program. HIV prevention for people living with HIV/AIDS: Tools for health care providers in HIV care and treatment settings. 2008. Retrieved from: http://positiveprevention.ucsf.edu/moz? page $=$ moz-CU04CDC4

Cloete, A.; Simbayi, L.; Strebel, A.; Mdleleni-Bookholane, T.; Josste, S.; Lewa, N. A public health evaluation of the healthy relationships positive prevention intervention programme in South Africa: Findings from a pilot study; Paper presented at the 9th international AIDS impact conference; 2009.

Cohen MS, Chen YQ, McCauley M, Gamble T, Hosseinipour MC, Kumarasamy N, et al. Prevention of HIV-1 infection with early antiretroviral therapy. New England Journal of Medicine. 2011; 365(6):493-505. [PubMed: 21767103]

Cornman DH, Kiene SM, Christie S, Fisher WA, Shuper PA, Pillay S, et al. Clinic-based intervention reduces unprotected sexual behavior among HIV-infected patients in KwaZulu-Natal, South Africa: Results of a pilot study. Clinical Science. 2008; 48(5):553-560.

Crepaz N, Lyles CM, Wolitski RJ, Passin WF, Rama SM, Herbst JH, et al. Do prevention interventions reduce HIV risk behaviours among people living with HIV? A meta-analytic review of controlled trials. AIDS. 2006; 20(2):143-157. [PubMed: 16511407]

Dewing S, Mathews C, Schaay N, Cloete A, Simbayi L, Chopra M. The feasibility of implementing a sexual risk reduction intervention in routine clinical practice at an ARV Clinic in Cape Town: A case study. AIDS and Behavior. 2010:1-6. [PubMed: 18843530]

Dodd PJ, Garnett GP, Hallett TB. Examining the promise of HIV elimination by 'Test and Treat' in hyper-endemic settings. AIDS. 2010; 24(5):729-735. [PubMed: 20154580]

Dolcini MM, Gandelman A, Vogan SA, Kong C, Leak TN, King AJ, et al. Translating HIV interventions into practice: Community-based organizations' experiences with the Diffusion of Effective Behavioral Interventions (DEBIs). Social Science \& Medicine. 2010; 71:1839-1846. [PubMed: 20926169]

Evangeli M, Engelbrecht SK, Swartz L, Turner K, Forsberg L, Soka N. An evaluation of a brief motivational interviewing training course for HIV/AIDS counsellors in Western Cape Province, South Africa. AIDS Care. 2009; 21(2):189-196. [PubMed: 19229688]

Ewart CK. Social Action Theory for a public health psychology. American Psychologist. 1991; 46(9): 931-946. [PubMed: 1958012]

Ewart, CK. Changing our unhealthy ways: Emerging perspectives from Social Action Theory. In: DiClemente, RJ.; Crosby, RA.; Kegler, MC., editors. Emerging theories in health promotion practice and research. San Francisco: Jossey-Bass; 2009. p. 359-391.

Fisher JD, Fisher WA, Cornman DH, Amico RK, Bryan A, Friedland GH. Clinician-delivered intervention during routine clinical care reduces unprotected sexual behavior among HIV-infected patients. Journal of Acquired Immune Deficiency Syndromes. 2006; 41(1):44-52. [PubMed: 16340472]

Galbraith JS, Stanton B, Boekeloo B, King W, Desmond S, Howard D, et al. Exploring implementation and fidelity of evidence-based behavioral interventions for HIV prevention: 
Lessons learned from the Focus on Kids diffusion case study. Health Education \& Behavior. 2009; 36(3):532-549. [PubMed: 18445739]

Gaydos CA, Hsieh YH, Galbraith JS, Barnes M, Waterfield G, Stanton B. Focus-on-Teens, sexual risk-reduction intervention for high-school adolescents: Impact on knowledge, change of riskbehaviours, and prevalence of sexually transmitted diseases. International Journal of STD \& AIDS. 2008; 19(10):704-710. [PubMed: 18824625]

Global Network of People Living with HIV, \& UNAIDS. Positive health, dignity and prevention. 2009

Holub, CK. Parental/caregiver influence on sexual risk behaviors among HIVpositive young people in Kinshasa, the Democratic Republic of the Congo. Chapel Hill: The University of North Carolina at Chapel Hill; 2010. Unpublished doctoral dissertation

Johnson MO, Charlebois E, Morin SF, Remien RH, Chesney MA. Effects of a behavioral intervention on antiretroviral medication adherence among people living with HIV: the Healthy Living Project randomized controlled study. Journal of Acquired Immune Deficiency Syndromes. 2007; 46(5): 574-580. [PubMed: 18193499]

Kalichman, S. Positive prevention: Reducing HIV transmission among people living with HIV/AIDS. New York: Springer; 2005.

Kalichman S, Rompa D, Cage M, DiFonzo K, Simpson D, Austin J, et al. Effectiveness of an intervention to reduce HIV transmission risks in HIV-positive people. American Journal of Prevention Medicine. 2001; 21(2):84-92.

Kelly JA, Heckman TG, Stevenson LY, Williams PN, Ertl T, Hays RB, et al. Transfer of researchbased HIV prevention interventions to community service providers: Fidelity and adaptation. AIDS Education and Prevention: Official Publication of the International Society for AIDS Education. 2000; 12(5 Suppl.):87. [PubMed: 11063072]

Lee MB, Leibowitz A, Rotheram-Borus MJ. Cost-effectiveness of a behavioral intervention for seropositive youth. AIDS Education and Prevention. 2005; 17(2):105-118. [PubMed: 15899749]

Lightfoot MA, Kasirye R, Comulada WS, Rotheram-Borus MJ. Efficacy of a culturally adapted intervention for youth living with HIV in Uganda. Prevention Science. 2007; 8(4):271-273. [PubMed: 17846891]

Lightfoot MA, Rotheram-Borus MJ, Tevendale H. An HIV-preventive intervention for youth living with HIV. Behavior Modification. 2007; 31(3):345-363. [PubMed: 17438347]

Luthar SS, Sawyer JA, Brown PJ. Conceptual issues in studies of resilience: Past, present, and future research. Annals of the New York Academy of Sciences. 2006; 1094(1):105-115. [PubMed: 17347344]

McKleroy VS, Galbraith JS, Cummings B, Jones P, Harshbarger C, Collins C, et al. Adapting evidence-based behavioral interventions for new settings and target populations. AIDS Education and Prevention. 2006; 18(4 Suppl. A):59-73. [PubMed: 16987089]

Ministry of Planning Democratic Republic of the Congo. HIV prevalence: Results from the Democratic Republic of the Congo 2007 Demographic and Health Survey. 2007

Montague B, Vuylsteke B, Buve A. Sustainability of programs to reach high risk and marginalized populations living with HIV in resource limited settings: Implications for HIV treatment and prevention. BMC Public Health. 2011; 11(1):701. [PubMed: 21917178]

Morin SF, Myers JJ, Shade SB, Koester K, Maiorana A, Rose CD. Predicting HIV transmission risk among HIV-infected patients seen in clinical settings. AIDS and Behavior. 2007; 11(5 Suppl):S6S16. [PubMed: 17577655]

Morrison DM, Hoppe MJ, Gillmore MR, Kluver C, Higa D, Wells EA. Replicating an intervention: The tension between fidelity and adaptation. AIDS Education and Prevention: Official Publication of the International Society for AIDS Education. 2009; 21(2):128. [PubMed: 19397435]

Noar SM. An interventionist's guide to AIDS behavioral theories. AIDS Care. 2007; 19(3):392-402. [PubMed: 17453575]

Parker L, Maman S, Pettifor A, Chalachala JL, Edmonds A, Golin C, et al. Feasibility analysis of an evidence-based positive prevention intervention for youth living with HIV/AIDS in Kinshasa, Democratic Republic of the Congo. 2012 submitted for publication. 
Peltzer K, Tabane C, Matseke G, Simbayi L. Lay counsellor-based risk reduction intervention with HIV positive diagnosed patients at public HIV counselling and testing sites in Mpumalanga, South Africa. Evaluation and Program Planning. 2010; 33(4):379-385. [PubMed: 20416948]

Pettifor, A.; Holub, C.; Mupenda, B.; Mujalambo, K.; Taylor, E.; Duvall, S., et al. Sexual behavior reported by HIV positive youth in Kinshasa, DRC confirms the need for positive prevention; Paper presented at the XVII international AIDS conference; 2008.

Poulsen MN, Vandenhoudt H, Wyckoff SC, Obong'o CO, Ochura J, Njika G, et al. Cultural adaptation of a US evidence-based parenting intervention for rural Western Kenya: From parents matter! To families matter! AIDS Education and Prevention. 2010; 22(4):273-285. [PubMed: 20707689]

Richardson JL, Milam J, McCutchan A, Stoyanoff S, Bolan R, Weiss J, et al. Effect of brief safer-sex counseling by medical providers to HIV-1 seropositive patients: A multi-clinic assessment. AIDS. 2004; 18(8):1179-1186. [PubMed: 15166533]

Rogers, EM. Diffusion of innovations. 4th ed. New York: The Free Press; 1995.

Rotheram-Borus, MJ. CLEAR: Choosing life: Empowerment, actions, results intervention manual. 1999. Retrieved from http://chipts.cch.ucla.edu/CLEAR

Rotheram-Borus, MJ. Together learning choices TLC intervention manual. 2001. Retrieved from http://chipts.cch.ucla.edu/tlc

Rotheram-Borus MJ, Ingram BL, Swendeman D, Flannery D. Common principles embedded in effective adolescent HIV prevention programs. AIDS and Behavior. 2009; 13(3):387-398. [PubMed: 19224358]

Rotheram-Borus, MJ.; Kelly, J.; Ehrhardt, AA.; Chesney, MA.; Lightfoot, M.; Weinhardt, L., et al. The NIMH Healthy Living Project. 2004. Retrieved from http://ari.ucsf.edu/programs/policy/ pwp_resources/HLP.ppt

Rotheram-Borus MJ, Lee MB, Murphy DA, Futterman D, Duan N, Birnbaum JM, et al. Efficacy of a preventive intervention for youths living with HIV. American Journal of Public Health. 2001; 91(3):400-405. [PubMed: 11236404]

Rotheram-Borus MJ, Swendeman D, Chovnick G. The past, present, and future of HIV prevention: Integrating behavioral, biomedical and structural intervention strategies for the next generation of HIV prevention. Annual Review of Clinical Psychology. 2009; 5:143-167.

Rotheram-Borus MJ, Swendeman D, Comulada WS, Weiss RE, Lee M, Lightfoot M. Prevention for substance-using HIV-positive young people: Telephone and in-person delivery. Journal of Acquired Immune Deficiency Syndromes. 2004; 37(Suppl. 2):S68-S77. [PubMed: 15385902]

Saleh-Onoya D, Braxton ND, Sifunda S, Reddy P, Ruiter R, Van den Borne B, et al. SISTA South Africa: The adaptation of an efficacious HIV prevention trial conducted with African-American women for isiXhosa-speaking South African women. Journal of Social Aspects of HIV/AIDS Research Alliance. 2008; 5(4):186-191. [PubMed: 19194600]

Saleh-Onoya D, Reddy PS, Ruiter RAC, Sifunda S, Wingood G, van den Borne B. Condom use promotion among isiXhosa speaking women living with HIV in the Western Cape Province, South Africa: A pilot study. AIDS Care. 2009; 21(7):817-825. [PubMed: 20024738]

The Healthy Living Project Team. Health-related interventions for persons living with HIV: Protocol. 1999.

The Healthy Living Project Team. Healthy Living Project Intervention Manual. Medical College of Wisconsino; 2004.

The Healthy Living Project Team. Effects of a behavioral intervention to reduce risk of transmission among people living with HIV: The Healthy Living Project randomized controlled study. Journal of Acquired Immune Deficiency Syndromes. 2007; 44(2):213-221. [PubMed: 17146375]

Tortolero SR, Markham CM, Parcel GS, Peters RJ, Escobar-Chaves SL, Basen-Engquist K, et al. Using intervention mapping to adapt an effective HIV, sexually transmitted disease, and pregnancy prevention program for high-risk minority youth. Health Promotion Practice. 2005; 6(3):286. [PubMed: 16020623]

UNAIDS. Global Report: UNAIDS report on the global AIDS epidemic 2010. 2010

Vandenhoudt H, Miller KS, Ochura J, Wyckoff SC, Obong'o CO, Otwoma NJ, et al. Evaluation of a US evidence-based parenting intervention in rural Western Kenya: From parents matter! To families matter! AIDS Education and Prevention. 2010; 22(4):328-343. [PubMed: 20707693] 
Wainberg ML, McKinnon K, Mattos PE, Pinto D, Mann CG, de Oliveira CSS, et al. A model for adapting evidence-based behavioral interventions to a new culture: HIV prevention for psychiatric patients in Rio de Janeiro, Brazil. AIDS and Behavior. 2007; 11(6):872-883. [PubMed: 17216334]

Walensky R. Cost-effectiveness of HIV interventions: From cohort studies and clinical trials to policy. International AIDS Society-USA. 2009; 17(4):130-134.

WHO. Essential prevention and care interventions for adults and adolescents living with HIV in resource-limited settings. Geneva: WHO; 2008.

WHO, UNAIDS, \& UNICEF. Progress report 2011 : Global HIV/AIDS response. 2011.

Wingood GM, DiClemente RJ. The ADAPT-ITT model: A novel method of adapting evidence-based HIV interventions. Journal of Acquired Immune Deficiency Syndromes. 2008; 47(Suppl. 1):S40 S46. [PubMed: 18301133]

Wingood GM, DiClemente RJ, Mikhail I, Lang DL, McCree DH, Davies SL, et al. A randomized controlled trial to reduce HIV transmission risk behaviors and sexually transmitted diseases among women living with HIV: The WiLLOW Program. Journal of Acquired Immune Deficiency Syndromes. 2004; 37(Suppl. 2):S58-S67. [PubMed: 15385901]

Wu Y, Stanton BF, Galbraith J, Kaljee L, Cottrell L, Li X, et al. Sustaining and broadening intervention impact: A longitudinal randomized trial of 3 adolescent risk reduction approaches. Pediatrics. 2003; 111(1):e32-e38. [PubMed: 12509592]

\section{Biographies}

Lisa Parker, PhD, Monitoring and Evaluation Technical Advisor, Futures Group, Chapel Hill, North Carolina, US.

Suzanne Maman, PhD, Associate Professor, Department of Health Behavior, Gillings School of Global Public Health, University of North Carolina, Chapel Hill, US.

Audrey Pettifor, PhD, Associate Professor, Department of Epidemiology, Gillings School of Global Public Health, University of North Carolina, Chapel Hill, US.

Jean Lambert Chalachala, MD, SYMPA Study Local Coordinator, Kinshasa School of Public Health, Kinshasa, The Democratic Republic of Congo.

Andrew Edmonds, PhD, Research Instructor, Gillings School of Global Public Health, Department of Epidemiology, University of North Carolina at Chapel Hill, Chapel Hill, US.

Carol Golin, MD, Associate Professor, Department of Health Behavior, Gillings School of Global Public Health, and Department of Medicine, University of North Carolina, Chapel Hill, US.

Kathryn Moracco, PhD, Research Associate Professor, Department of Health Behavior, Gillings School of Global Public Health, University of North Carolina, Chapel Hill, US.

Frieda Behets, PhD, Professor, Department of Epidemiology, Gillings School of Global Public Health, Research Professor, Department of Medicine, University of North Carolina, Chapel Hill, US. 


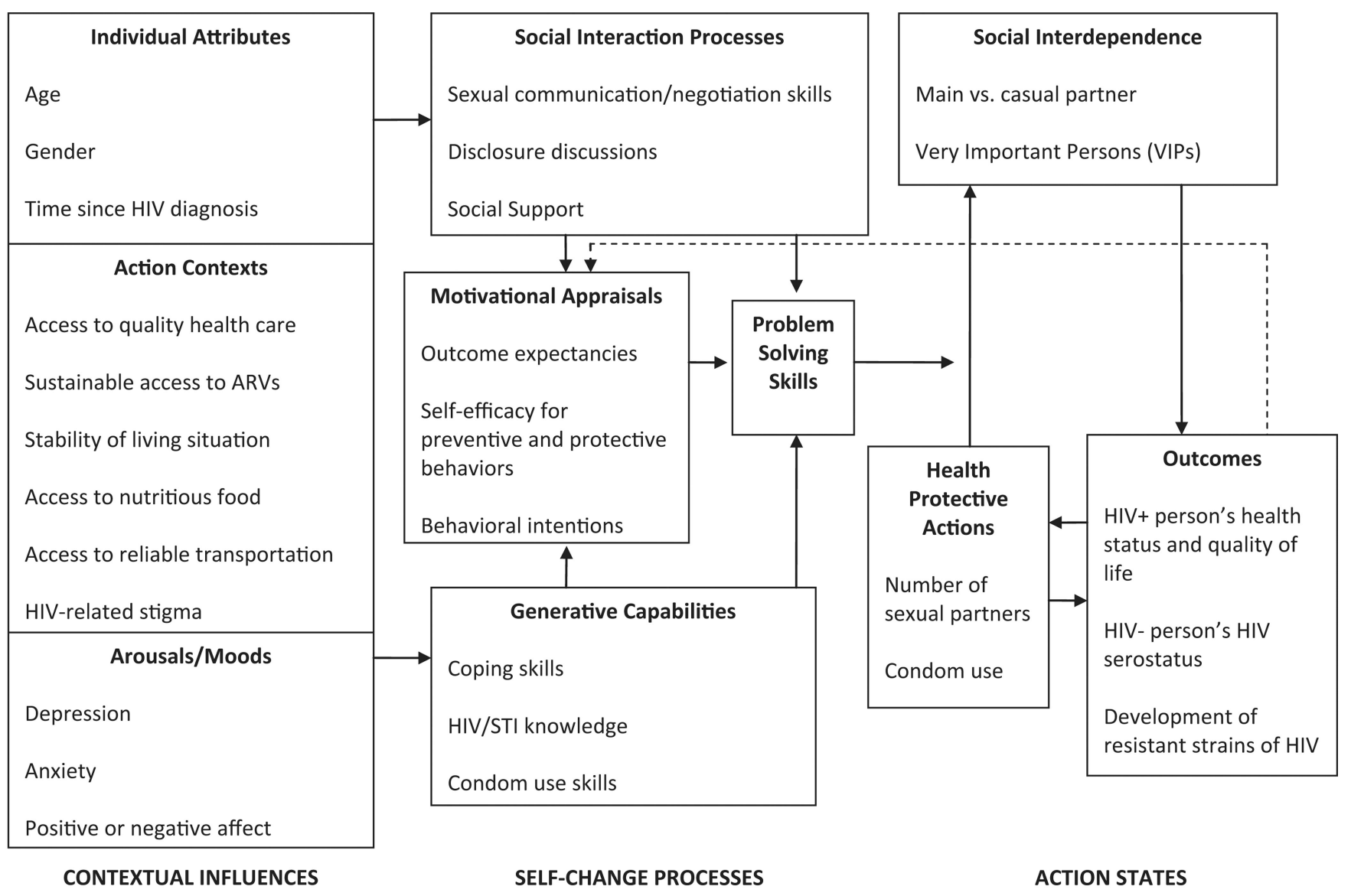

Source: Adapted from (Ewart, 1991; M. Lightfoot, Rotheram-Borus, \&Tevendale, 2007)

Fig. 1.

Conceptual framework for SYMPA. 
Table 1

Assessment of EBIs considered for adaptation for YLWH in Kinshasa, DRC.

\begin{tabular}{ll}
\hline Healthy relationships \\
\\
\\
\hline Core & Defining stress and reinforcing \\
elements & coping skills, disclosing \\
to family and friends, disclosing to & sexual partners, and \\
& building healthier and safer \\
& relationships. \\
& Using modeling, role play, and \\
& feedback to teach and \\
& practice skills related to coping \\
& with stress. \\
& Teaching decision making skills \\
& about disclosure of HIV \\
& status. \\
& Providing personal feedback \\
& reports to motivate change \\
& of risky behaviors and continuance \\
& of protective \\
& behaviors. \\
& Using movie clips to set up \\
scenarios about disclosure \\
and risk reduction to stimulate \\
discussions and role \\
plays.
\end{tabular}

\section{Choosing Life: \\ Empowerment, \\ Actions, \\ Results (CLEAR)}

Development of emotional awareness

through use of a Feeling

Thermometer

and identification of the

link among

feelings, thoughts, and

actions.

Identification of Ideal

Self to help

motivate and personalize

behavior

change.

Teaching, modeling, and

practicing

short and long term goal

setting.

Teaching, modeling, and

practicing

SMART problem

solving.

Teaching, modeling, and

practicing

assertive behavior and

communication.
Key characteristics Have clients meet in small groups (five to 12 people).

New members cannot join once the series of sessions has

begun.

Have clients sit face to face in a circle.

Have clients meet for at least five sessions, $2 \mathrm{~h}$ each.

Ensure that groups contain members of the same gender and sexual orientation.

Have two facilitators per group.

Ensure that facilitators are of both

genders, experienced

counselors, HIV-infected if

possible, and same ethnicity of clients.
Use incentives to encourage clients to

return to sessions.

Complete each session

in 60-75 min.

Conduct sessions once

per week to

allow clients time to

practice new skills

before next session.

Use experienced

counselors or mental

health professionals who

have received

formal clinical training

Deliver the intervention

with a client-

centered approach

Hold sessions in room

that ensures

privacy and

confidentiality and is

free

from interruptions.

\section{Together Learning The Healthy Living \\ Choices (TLC) $\quad$ Project $^{a}$}

Help clients develop Core repertoire of cognitive-

awareness and

identify feelings,

thoughts and actions

Teach, model, and practice emotional regulation, SMART problem solving, goal setting, and assertiveness. Reinforce positive client behavior through the use of thanks tokens. Help clients identify

heir Ideal Self to help motivate and personalize behavior change.

Deliver sessions in highly participatory, interactive small groups.

Encourage clients to attend all sessions,

but give them

flexibility to drop in for

particular sessions on

their own

schedule.

Consider use of

ncentives and

providing food/

snacks.

Conduct sessions about once a week, limit session times, and modify time

intervals.

Let group needs determine facilitators

Be flexible about group composition, start with small groups, and build group

cohesion

Use visual aids.

Consider your

location. behavioral techniques

covered in each session,

including trigger

identification,

problem solving, and goal

setting.

Content of each session will need to be tailored to the life-context of individual participants.

Focus on addressing quality of life, psychologic coping, achieving positive affect and supportive social relationships.

Focus on addressing self regulatory issues, such as avoiding sexual and drugrelated risk of HIV

transmission or acquisition of additional sexually

transmitted diseases, and disclosure of HIV status to potential partners.

Focus on addressing accessing health services, medication adherence, and active participation in medical care decision making.

Sessions occur about every week.

Each module separated from the next by three months to allow participants time to incorporate the information from the program into their lives and to evaluate impact of each module on their wellbeing.

Facilitator as coach who helps the participants achieve

goals and make changes in

their lives.

Participants received

incentives for each module. Fifteen 90-min individual counseling sessions.

Core activities to be

delivered as active part of program,

open to be tailored to each participant, and beginning and end of each session to be adapted to each participant. 


\begin{tabular}{|c|c|c|c|c|}
\hline & Healthy relationships & $\begin{array}{l}\text { Choosing Life: } \\
\text { Empowerment, } \\
\text { Actions, } \\
\text { Results (CLEAR) }\end{array}$ & $\begin{array}{l}\text { Together Learning } \\
\text { Choices (TLC) }\end{array}$ & $\begin{array}{l}\text { The Healthy Living } \\
\text { Project }^{a}\end{array}$ \\
\hline Delivery methods & $\begin{array}{l}\text { Exercises } \\
\text { Goal setting } \\
\text { Group discussions } \\
\text { Lectures } \\
\text { Role plays } \\
\text { Printed materials } \\
\text { Video } \\
\text { Coping strategies }\end{array}$ & $\begin{array}{l}\text { Exercise/games } \\
\text { Goal setting } \\
\text { Demonstration } \\
\text { Lectures } \\
\text { Role plays } \\
\text { Practice }\end{array}$ & $\begin{array}{l}\text { Exercises } \\
\text { Goal setting } \\
\text { Group discussion } \\
\text { Practice } \\
\text { Role plays } \\
\text { Video } \\
\text { Problem-solving }\end{array}$ & $\begin{array}{l}\text { Exercises } \\
\text { Coping strategies } \\
\text { Goal setting } \\
\text { Demonstration } \\
\text { Problem-solving } \\
\text { Role plays }\end{array}$ \\
\hline Theoretical basis & Social Cognitive Theory & $\begin{array}{l}\text { Cognitive Behavior } \\
\text { Therapy } \\
\text { Social Action Theory }\end{array}$ & $\begin{array}{l}\text { Social Action Theory } \\
\text { Social Cognitive } \\
\text { Theory }\end{array}$ & $\begin{array}{l}\text { Social Action Theory } \\
\text { Social Cognitive Theory }\end{array}$ \\
\hline Goodness of fit & $\begin{array}{l}\text { Pronounced focus on self-efficacy } \\
\text { for disclosure - not } \\
\text { determined to be a major } \\
\text { determinant identified in } \\
\text { target population. } \\
\text { Least comprehensive. } \\
\text { Did not include option for } \\
\text { participants who were } \\
\text { abstinent or not ever had sex - thus } \\
\text { not appropriate for } \\
\text { youth population. }\end{array}$ & $\begin{array}{l}\text { Substance abuse highly } \\
\text { integrated } \\
\text { within entire } \\
\text { intervention - not } \\
\text { deemed a good fit with } \\
\text { target } \\
\text { population needs. }\end{array}$ & $\begin{array}{l}\text { Combined multiple } \\
\text { determinants and } \\
\text { behaviors in same } \\
\text { module - determined } \\
\text { to be too complex for } \\
\text { target population. }\end{array}$ & $\begin{array}{l}\text { Used less sexually explicit } \\
\text { language than CLEAR or } \\
\text { TLC. } \\
\text { Specific case studies } \\
\text { provided for participants not } \\
\text { sexually active. } \\
\text { Most comprehensive and } \\
\text { flow and progression of } \\
\text { session and activities best } \\
\text { organized. } \\
\text { Sessions user-friendly and } \\
\text { more easily adaptable. }\end{array}$ \\
\hline
\end{tabular}

Source: Adapted from CDC Best-Evidence website http://www.cdc.gov/hiv/topics/research/prs/best-evidence-intervention.htm (CDC, 2012a), Diffusion of Effective Behavioral Intervention website http://www.effectiveinterventions.org/en/home.aspx (AED, 2011), and Intervention Manuals (CDC, 2012b; Rotheram-Borus, 1999, 2001; The Healthy Living Project Team, 2004).

${ }^{a}$ Core elements and key characteristics for the Healthy Living Project have not yet been established as the intervention is not part of the CDC Diffusion of Effective Behavioral Intervention project. Therefore information was adapted from the Healthy Living Project Intervention Manual and published articles. Interestingly, the primary investigators for the HLP have published extensively on the need for "common factors" and "common processes" and "common principles" rather than core elements and key characteristics (Johnson, Charlebois, Morin, Remien, \& Chesney, 2007; Rotheram-Borus, Swendeman, et al., 2009). 
Table 2

Healthy Living Project Intervention overview.

\begin{tabular}{|c|c|}
\hline Session & Content \\
\hline \multicolumn{2}{|l|}{ Module 1: Stress, coping, and adjustment } \\
\hline 1. Orientation and overview & Preview intervention and identify personal strengths and challenges; setting attainable goal \\
\hline 2. Stress and coping I & Identify personal stressors; changeable vs. unchangeable stressors; problem solving skills \\
\hline 3. Stress and coping II & Problem-focused vs. emotion-focused coping; matching coping to stressing type \\
\hline 4. Social support & $\begin{array}{l}\text { Three types of social support (emotional, informational, and tangible); identify ways to } \\
\text { increase positive support }\end{array}$ \\
\hline 5. Adaptive coping & Develop plan to continue decreasing stress by increasing adaptive coping strategies \\
\hline \multicolumn{2}{|l|}{ Module 2: Safer behaviors } \\
\hline 1. Knowledge & Risk continuum; identify personal risk limits and problem solve triggers to risky behavior \\
\hline 2. Safer behavior & Proper condom use; knowledge of STIs and needle exchange programs \\
\hline 3. Assertive communication/negotiation skills & $\begin{array}{l}\text { Components of assertive communication; role plays to increase communication/negotiation } \\
\text { skills }\end{array}$ \\
\hline 4. Disclosure decisions & Identify person and environmental factors related to disclosure decisions \\
\hline 5. Keeping safe & $\begin{array}{l}\text { Maintaining/increasing safer behaviors, assertive communication/negotiation skills, and } \\
\text { disclosure self-efficacy }\end{array}$ \\
\hline \multicolumn{2}{|l|}{ Module 3: Health behaviors } \\
\hline 1. Current health behaviors & Identify factors that influence medical regimen adherence \\
\hline 2. Maintenance & Assertive communication with health care providers and others who influence adherence \\
\hline 3. Social support and adherence & Problem solve challenges to self-efficacy and to obtaining social support for adherence \\
\hline 4. Staying on track & Develop plan for maintaining adherence \\
\hline 5. Future choices & Identify successes and problem solve challenges to maintain behavioral and attitudinal changes \\
\hline
\end{tabular}

Source: Johnson et al. (2007). 
Table 3

SYMPA adaptations to the Healthy Living Project core elements, key characteristics, delivery methods, and theoretical basis.

\begin{tabular}{|c|c|c|}
\hline & The Healthy Living Project $a$ & SYMPA - adaptations \\
\hline Core elements & $\begin{array}{l}\text { Core repertoire of cognitive-behavioral techniques covered in each session, } \\
\text { including trigger identification, problem solving, and goal setting. } \\
\text { Content of each session will need to be tailored to the life context of } \\
\text { individual } \\
\text { participants. } \\
\text { Focus on addressing quality of life, psychologic coping, achieving positive } \\
\text { affect and } \\
\text { supportive social relationships. } \\
\text { Focus on addressing self-regulatory issues, such as avoiding sexual and } \\
\text { drug- } \\
\text { related risk of HIV transmission or acquisition of additional sexually } \\
\text { transmitted } \\
\text { diseases, and disclosure of HIV status to potential partners. } \\
\text { Focus on addressing accessing health services, medication adherence, and } \\
\text { active } \\
\text { participation in medical care decision making. }\end{array}$ & $\begin{array}{l}\text { No changes. } \\
\text { Sessions tailored to life context of } \\
\text { individual } \\
\text { participants but in the context of group } \\
\text { delivery. } \\
\text { No changes. } \\
\text { Drug-related content removed. } \\
\text { This module was removed. }\end{array}$ \\
\hline Key characteristics & $\begin{array}{l}\text { Sessions occur about every week. } \\
\text { Each module separated from the next by three months to allow participants } \\
\text { time to } \\
\text { incorporate the information from the program into their lives and to } \\
\text { evaluate } \\
\text { impact of each module on their wellbeing. } \\
\text { Facilitator as coach who helps the participants achieve goals and make } \\
\text { changes in } \\
\text { their lives. } \\
\text { Participants received incentives for each module. } \\
\text { Fifteen } 90 \text {-min individual counseling sessions. } \\
\text { Core activities to be delivered as active part of program, open to be tailored } \\
\text { to each } \\
\text { participant, and beginning and end of each session to be adapted to each } \\
\text { participant. }\end{array}$ & $\begin{array}{l}\text { No changes. } \\
\text { Sessions occurred every week for six } \\
\text { weeks. } \\
\text { No changes. } \\
\text { No changes. } \\
\text { Original } 15 \text { sessions reduced to six. } \\
\text { Sessions open to be tailored to each } \\
\text { participant } \\
\text { but in the context of group delivery. }\end{array}$ \\
\hline Delivery methods & $\begin{array}{l}\text { Exercises } \\
\text { Coping strategies } \\
\text { Goal setting } \\
\text { Demonstration } \\
\text { Problem solving } \\
\text { Role plays }\end{array}$ & No changes. \\
\hline Theoretical basis & $\begin{array}{l}\text { Social Action Theory } \\
\text { Social Cognitive Theory }\end{array}$ & No changes. \\
\hline
\end{tabular}

Source: Adapted from CDC Best-Evidence website http://www.cdc.gov/hiv/topics/research/prs/best-evidence-intervention.htm (CDC, 2012a), Diffusion of Effective Behavioral Intervention website http://www.effectiveinterventions.org/en/home.aspx (AED, 2011), and Intervention Manuals (CDC, 2012b; Rotheram-Borus, 1999, 2001; The Healthy Living Project Team, 2004).

${ }^{a}$ Core elements and key characteristics for the Healthy Living Project have not yet been established as the intervention is not part of the CDC Diffusion of Effective Behavioral Intervention project. Therefore information was adapted from the Healthy Living Project Intervention Manual and published articles. 
Table 4

SYMPA intervention session content overview.

\begin{tabular}{|c|c|c|}
\hline Session number & Session title & Content \\
\hline Session 1 & Living with HIV & Preview intervention and identify personal strengths and challenges; setting attainable goal \\
\hline Session 2 & Coping and problem solving & $\begin{array}{l}\text { Identify personal stressors; changeable vs. unchangeable stressors; problem solving skills; } \\
\text { problem- } \\
\text { focused vs. emotion-focused coping; matching coping to stressing type. }\end{array}$ \\
\hline Session 3 & Safer Sex I & Risk continuum; identify personal risk limits and problem solve triggers to risky behavior. \\
\hline Session 4 & Safer Sex II & $\begin{array}{l}\text { Proper condom use; knowledge of STIs and needle exchange programs; components of } \\
\text { assertive } \\
\text { communication; role plays to increase communication/negotiation skills. }\end{array}$ \\
\hline Session 5 & $\begin{array}{l}\text { Social Support and } \\
\text { Disclosure }\end{array}$ & $\begin{array}{l}\text { Three types of social support (emotional, informational, and tangible); identify ways to } \\
\text { increase positive } \\
\text { support; identify person and environmental factors related to disclosure decisions. Youth } \\
\text { identify Very } \\
\text { Important Person (VIP) in their support network who has been supportive and who could } \\
\text { assist them after } \\
\text { the intervention ends with maintaining healthy behaviors. }\end{array}$ \\
\hline Session 6 & $\begin{array}{l}\text { Wellbeing and Health } \\
\text { Maintenance }\end{array}$ & $\begin{array}{l}\text { Identify successes and problem solve challenges to maintain behavioral and attitudinal } \\
\text { changes. Focus on } \\
\text { developing a plan to maintain healthy behaviors after the intervention ends and the role of } \\
\text { the VIP in } \\
\text { supporting the youth in this process. }\end{array}$ \\
\hline
\end{tabular}

